\title{
Reason of Discontinuation After Transarterial Chemoembolization Influences Survival in Patients with Hepatocellular Carcinoma
}

\author{
Tim A. Labeur ${ }^{1,2} \cdot$ R. Bart Takkenberg ${ }^{3}$ Heinz-Josef Klümpen ${ }^{1,2}$ • \\ Otto M. van Delden 4
}

Received: 30 August 2018/Accepted: 8 November 2018/Published online: 28 November 2018

(C) The Author(s) 2018

\begin{abstract}
Background Transarterial chemoembolization (TACE) for intermediate-stage hepatocellular carcinoma (HCC) is often repeated until unTACEable progression (UTP) occurs. There is little data on the various reasons for stopping TACE and its consequences for subsequent treatment and survival.

Aim To assess the impact of the various reasons of UTP on survival and consequences for subsequent treatments.

Methods Consecutive HCC patients who underwent TACE between 2003 and 2016 were analyzed retrospectively for the reason of TACE discontinuation. UTP was defined
\end{abstract}

Electronic supplementary material The online version of this article (https://doi.org/10.1007/s00270-018-2118-6) contains supplementary material, which is available to authorized users.

Otto M. van Delden

o.m.vandelden@amc.nl

Tim A. Labeur

t.a.labeur@amc.nl

R. Bart Takkenberg

r.b.takkenberg@amc.nl

Heinz-Josef Klümpen

h.klumpen@amc.nl

Cancer Center Amsterdam, Amsterdam, The Netherlands

2 Department of Medical Oncology, Amsterdam University Medical Centers, University of Amsterdam, Meibergdreef 9, Amsterdam, The Netherlands

3 Department of Gastroenterology and Hepatology, Amsterdam University Medical Centers, University of Amsterdam, Meibergdreef 9, Amsterdam, The Netherlands

4 Department of Radiology and Nuclear Medicine, Amsterdam University Medical Centers, University of Amsterdam, Meibergdreef 9, 1105 AZ Amsterdam, The Netherlands according to the EASL guidelines, considering radiological pattern of progression, liver function and performance status (PS). Overall and post-progression survival (OS, PPS) for different reasons of TACE discontinuation were compared. The correlation between time to untreatable progression by chemoembolization (TTUPc) and OS was analyzed.

Results One hundred and sixty-six patients (BCLC-A 40\%, BCLC-B 54\%, BCLC-C: 7\%) were included, undergoing a median of 2 TACE procedures with a median OS of 22.1 months (95\% CI 17.4-26.7). UTP occurred in 116 patients (70\%) after a median TTUPc of 11.6 months (95\% CI 7.8-15.4). There was a strong positive correlation ( $\rho=0.816, p<0.001)$ between TTUPc and OS. The main reason of UTP was radiological progression (61\%), which was mostly intrahepatic (75\%). Hepatic decompensation and worsening of PS were independent predictors of OS and PPS.

Conclusion The majority of HCC patients treated with TACE have UTP due to intrahepatic tumor progression with preserved liver function and PS, making them potential candidates for subsequent liver-directed or systemic treatment. TTUPc may be a valuable surrogate endpoint for OS in patients treated with TACE.

Level of Evidence Level II, prognosis study.

Keywords Hepatocellular carcinoma - Transarterial chemoembolization - TACE - Discontinuation - Time to untreatable progression by chemoembolization .

Survival

$\begin{array}{ll}\text { Abbreviations } \\ \text { AFP } & \text { Alpha-fetoprotein } \\ \text { BCLC } & \text { Barcelona Clinic Liver Cancer } \\ \text { BSC } & \text { Best supportive care }\end{array}$

Abbreviations

AFP Alpha-fetoprotein

BSC Best supportive care 


\begin{tabular}{|c|c|}
\hline CT & Computed tomography \\
\hline cTACE & $\begin{array}{l}\text { Conventional } \\
\text { chemoembolization }\end{array}$ \\
\hline DEB-TACE & $\begin{array}{l}\text { Drug-eluting beads transarterial } \\
\text { chemoembolization }\end{array}$ \\
\hline EASL & $\begin{array}{l}\text { European Association for the Study of the } \\
\text { Liver }\end{array}$ \\
\hline ECOG PS & $\begin{array}{l}\text { Eastern Cooperative Oncology Group } \\
\text { performance status }\end{array}$ \\
\hline EHS & Extrahepatic spread \\
\hline HBV & Hepatitis B virus \\
\hline $\mathrm{HCC}$ & Hepatocellular carcinoma \\
\hline $\mathrm{HCV}$ & Hepatitis $C$ virus \\
\hline IQR & Interquartile range \\
\hline MDT & Multidisciplinary team \\
\hline mRECIST & $\begin{array}{l}\text { Modified response evaluation criteria in } \\
\text { solid tumors }\end{array}$ \\
\hline MRI & Magnetic resonance imaging \\
\hline MVI & Macrovascular invasion \\
\hline OS & Overall survival \\
\hline PD & Progressive disease \\
\hline PPS & Post-progression survival \\
\hline $\mathrm{RE}$ & Radioembolization \\
\hline RFA & Radiofrequency ablation \\
\hline TACE & Transarterial chemoembolization \\
\hline TTUPc & $\begin{array}{l}\text { Time to untreatable progression } \\
\text { chemoembolization }\end{array}$ \\
\hline UTP & unTACEable progression \\
\hline
\end{tabular}

\section{Introduction}

Transarterial chemoembolization (TACE) currently is the cornerstone of treatment for patients with intermediatestage hepatocellular carcinoma (HCC) [1, 2], based on two randomized controlled trials showing survival benefit of TACE compared with best supportive care (BSC) in patients with unresectable HCC [3, 4]. The beneficial effect of TACE was confirmed by two meta-analyses [5, 6]. However, with the expanding number of loco-regional (radioembolization) and systemic treatments (sorafenib [7], lenvatinib [8], regorafenib [9], cabozantinib [10], ramucirumab [11] and nivolumab [12]) for HCC, the guidelineendorsed concept of timely switch to alternative treatments [1], or treatment stage migration, is becoming increasingly relevant.

TACE can be performed repeatedly, but the potential survival benefit of each TACE should be carefully weighed against the risk of damaging normal hepatocytes and worsening liver function which may preclude subsequent treatments and potentially impair survival outcomes [13]. This has led to several scoring systems designed to identify the best candidates for TACE in order to maximize treatment benefit and to select candidates for alternatives therapies [14]. Current guidelines have not yet endorsed these predictive models, but underscore the importance of switching to alternative treatments in case of 'unTACEable progression' (UTP) [1]. The definition of UTP is based on expert opinion and varies between centers and countries [15-17]. Most definitions include radiological progression and deterioration of liver function or performance status. There is limited data on the impact of the various reasons for UTP in clinical practice, although this strongly influences the choice for a next-line treatment. The selection of a subsequent treatment is often based on the radiological pattern of tumor progression after TACE, i.e., progression of intrahepatic lesions, appearance of macrovascular invasion (MVI) or extrahepatic spread (EHS). Radiological pattern of progression has been shown to impact postprogression survival of HCC patients treated with sorafenib [18]. Prior studies have reported the incidence of tumor progression [3, 4] and pattern of tumor recurrence following TACE [19-21], but data on the prognostic impact of pattern of tumor progression following TACE are lacking. Lastly, the advent of multiple lines of subsequent treatments is making it increasingly difficult to assess the benefit of TACE based on overall survival (OS) alone. Novel surrogate outcomes such as time to untreatable progression by chemoembolization (TTUPc) $[15,22]$ have been proposed as a useful parameter for treatment guidance and valuable endpoint of future trials involving TACE. However, TTUPc requires validation in clinical practice.

This retrospective study of patients with HCC treated with TACE aims to (1) analyze the reason of UTP and radiological pattern of tumor progression and assess the impact on subsequent treatments and survival outcomes, (2) determine whether TTUPc is a useful surrogate parameter for estimating TACE benefit in terms of OS.

\section{Methods}

\section{Study Population}

This retrospective study was approved by the Institutional Review Board, and the need for informed consent was waived (reference number W17_420\#17.488).

From February 2003 to November 2016, consecutive patients with liver-confined HCC and preserved liver function (Child-Pugh $\leq$ B7) who underwent TACE at our tertiary referral hospital were included. Patients were 
identified by querying the electronic patient registration systems and the institutional radiology archive.

\section{Diagnostic Workup and Treatment Algorithm}

HCC was diagnosed by pathology or imaging criteria according to the European Association for the Study of the Liver (EASL) guidelines [1]. All patients were staged with 4-phase contrast-enhanced computed tomography (CT) or magnetic resonance imaging (MRI) and discussed in the HCC multidisciplinary team (MDT). Patients were considered for TACE according to the Barcelona Clinic Liver Cancer (BCLC) algorithm $[1,23]$. Accordingly, TACE was considered in patients with BCLC-B, or those with BCLC$A$ in whom surgery or radiofrequency ablation (RFA) was not deemed possible. Patients with portal vein invasion (BCLC-C) only were considered for TACE, if tumor invasion was limited to segmental portal veins.

TACE was performed using the standard technique as described previously [24]. Before 2008, conventional TACE (cTACE) was performed using an emulsion of doxorubicin $\left(50-75 \mathrm{mg} / \mathrm{m}^{2}\right)$ and lipiodol followed by gelatin sponge. Since 2008, patients underwent TACE with drug-eluting beads (DEB-TACE) loaded with doxorubicin (75-150 mg)(DC beads 100-300 $\mu \mathrm{m}$, Terumo Europe, Belgium). Patients undergoing either cTACE or DEBTACE were evaluated in this study as both techniques have similar survival benefit [25]. Follow-up after single- or multi-session TACE included clinical, biochemical and radiological assessment after 6 weeks and every 3 subsequent months. Radiological response was assessed by multiphasic CT or MRI using the modified response evaluation criteria in solid tumor (mRECIST) criteria [14]. All patients were re-evaluated in the MDT after each follow-up visit, and additional TACE was performed in cases of noncomplete response or appearance of intrahepatic recurrence. At UTP, patients were considered for subsequent treatment including sorafenib $(\geq 2008)$ and radioembolization $(\geq 2012)$.

\section{Outcomes}

According to the EASL guidelines, UTP is a clinical profile that prohibits further TACE treatment [1], defined as: radiological tumor progression, including intrahepatic growth or non-response of target lesions after $\geq 2$ TACE, or occurrence of extrahepatic spread (EHS) or macrovascular invasion (MVI); hepatic decompensation (ChildPugh $\geq \mathrm{B} 8$, uncontrolled ascites or encephalopathy); or worsening of performance to Eastern Cooperative Oncology Group performance status (ECOG PS) $>2$. In case of radiological tumor progression, this was further specified according to radiological pattern of progression. OS was measured from date of first TACE to date of death or last known date to be alive. Survival status was checked using the municipal records database on May 4, 2018. When TACE was used as bridging treatment to curative resection or liver transplantation, OS was censored on date of surgery. OS was divided into time to untreatable progression by chemoembolization (TTUPc) and post-progression survival (PPS) as proposed by Kudo et al. [16] (Supplementary Fig. 1). TTUPc was defined from date of first TACE to date of UTP or censored at the time of last radiological evaluation. Patients without UTP who did not have at least one radiological evaluation were excluded from TTUPc analysis. PPS was defined as the period from date of UTP to date of death or last follow-up.

The OS and PPS were compared according to different reasons of UTP. Moreover, the radiological pattern of progression was analyzed in a subgroup of patients with preserved liver function and ECOG PS, thereby eliminating the competing risk of impairment of ECOG PS and liver function.

\section{Statistical Analysis}

Categorical variables were described as frequencies with percentages and continuous variables as medians with interquartile ranges (IQR). Time-to-event data were estimated by Kaplan-Meier method with plot and median (95\% confidence interval $[95 \% \mathrm{CI}]$ ). Differences in survival rate were assessed by log-rank test. To assess the association between survival (OS and PPS) and the reason of UTP and radiological pattern, these were analyzed in a multivariable Cox proportional hazards analysis, adjusting for known prognostic factors [26] and additional factors that were associated with survival in univariable analysis $(p<0.1)$. Hazard ratios (HR) with $95 \%$ confidence intervals (CI) were calculated. The relationship between TTUPc/PPS and OS was assessed with the Spearman correlation $(\rho)$ test in the whole cohort. For all statistical tests, a two-tailed $p$ value of $<0.05$ was considered statistically significant. All analyses were performed using IBM SPSS Statistics for Windows Version 24.0 (IBM Corp., Armonk, NY, USA).

\section{Results}

\section{Patient Characteristics Prior to First TACE}

Between February 2003 and November 2016, 197 patients who underwent TACE for HCC were identified at our institution. After exclusion of 31 patients, 166 patients met the eligibility criteria and formed the study cohort (Fig. 1). The baseline demographic, clinical and imaging 
characteristics are summarized in Table 1. Prior to TACE, $88(54 \%)$ patients had intermediate-stage HCC (BCLC-B) and $54(40 \%)$ patients had early-stage HCC (BCLC-0/A) which was treated with TACE due to ineligibility for liver resection, transplantation or local ablation. Eleven (7\%) patients had advanced stage (BCLC-C), due to segmental MVI.

\section{Treatment Details and Reason of unTACEable Progression}

Patients underwent a median of 2 TACE sessions (range 1-7), mostly DEB-TACE (78\%). Treatment details are summarized in Supplementary Table 1. At the time of database lock, May 4, 2018, 50 patients did not have UTP, for example, due to complete remission or liver transplantation after TACE, or early TACE cessation (Figure 1). Consequently, 116 patients developed UTP. Most of these had radiological tumor progression $(n=105$, $91 \%$ ), sometimes in combination with deteriorated ECOG PS $(n=12,7 \%)$ or hepatic decompensation $(n=22,19 \%)$. The radiological pattern of tumor progression is specified in detail in Fig. 1, including in intrahepatic progression $(n=61,58 \%)$, intrahepatic progression with MVI $(n=18$, $17 \%)$ and EHS $(n=26,25 \%)$. When considering all $(n=27)$ patients with hepatic decompensation at the time of TACE discontinuation, with $(n=22)$ or without $(n=5)$ tumor progression, only $3 / 27(11 \%)$ patients recovered to Child-Pugh $\leq \mathrm{B} 7$ rendering them potentially eligible for subsequent treatment.

After UTP, subsequent treatment was given in 58 patients, mainly sorafenib $(n=42)$ or radioembolization

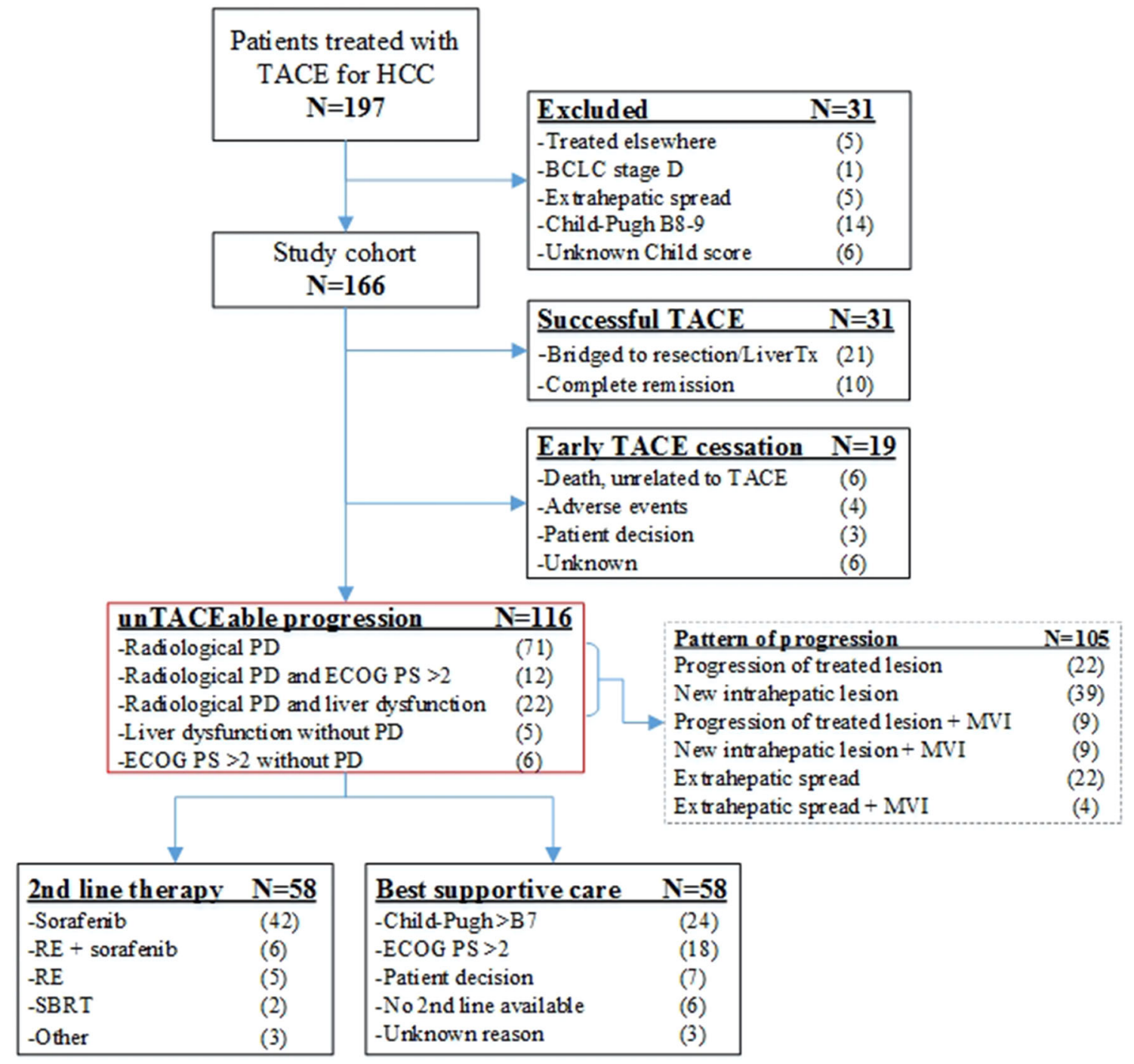

Fig. 1 Consort flow diagram. $B C L C$ Barcelona Clinic Liver Cancer; ECOG PS Eastern Cooperative Oncology Group performance status; $H C C$ hepatocellular carcinoma; LiverTx liver transplantation; MVI macrovascular invasion; $P D$ progressive disease; $R E$ radioembolization; SBRT stereotactic body radiotherapy (SBRT); TACE transarterial chemoembolization 
Table 1 Baseline characteristics of patients (prior to first TACE)

\begin{tabular}{|c|c|}
\hline Characteristic & All patients $(N=166)$ \\
\hline Age-years (IQR) & $69(61-74)$ \\
\hline Males-n $(\%)$ & $129(78)$ \\
\hline \multicolumn{2}{|l|}{ Etiology-n $(\%)$} \\
\hline Alcohol & $63(38)$ \\
\hline $\mathrm{HBV}$ & $24(15)$ \\
\hline $\mathrm{HCV}$ & $53(32)$ \\
\hline NAFLD/NASH & $16(10)$ \\
\hline Other/unknown & $10 / 19(6 / 11)$ \\
\hline Cirrhosis-n $(\%)$ & $146(89)$ \\
\hline \multicolumn{2}{|l|}{ Child-Pugh class- $n(\%)$} \\
\hline A/B7 & 133/13 (91/9) \\
\hline \multicolumn{2}{|l|}{ ECOG PS- $n(\%)$} \\
\hline $0 / 1 / 2$ & $94 / 58 / 12(57 / 35 / 7)$ \\
\hline \multicolumn{2}{|l|}{ BCLC stage- $n(\%)$} \\
\hline 0 or $\mathrm{A} / \mathrm{B} / \mathrm{C}$ & $67 / 88 / 11(40 / 53 / 7)$ \\
\hline \multicolumn{2}{|l|}{ Number of tumor nodules- $n(\%)$} \\
\hline $1 / 2-3 />3$ & $54 / 68 / 44(33 / 41 / 27)$ \\
\hline Size of largest nodule-mm (IQR) & $46(34-61)$ \\
\hline Macroscopic vascular invasion- $n(\%)$ & $11(7)$ \\
\hline
\end{tabular}

BCLC Barcelona Clinic Liver Cancer classification; ECOG PS Eastern Cooperative Oncology Group performance status; $H B V$ hepatitis B virus; $H C V$ hepatitis $\mathrm{C}$ virus; $I Q R$ interquartile range; NAFLD/NASH non-alcoholic fatty liver disease/non-alcoholic steatohepatitis; TACE transarterial chemoembolization

$(n=11)$. Patients who received best supportive care (BSC, $n=58)$ often had hepatic decompensation $(n=24)$ or ECOG PS $>2(n=18)$ prohibiting treatment. Thirteen patients did not receive subsequent treatment despite eligibility, i.e., due to patient decision or unavailability of a subsequent line of treatment $(<2008)$.

\section{Overall Survival, Time to unTACEable Progression and Post-Progression Survival}

After a median follow-up of 40.5 months $(95 \% \mathrm{CI}$ 27.6-53.3), 115 out of 166 (69\%) patients had died. The median OS was 22.1 months (95\% CI 17.4-26.7). One patient did not have $\geq 1$ imaging evaluation; thus, 165 patients were available for TTUPc analysis. UTP occurred in 116 out of 165 patients (70\%) after a median TTUPc of 13.3 months (95\% CI 10.4-16.3). The median PPS and OS in patients who had UTP $(n=116)$ was 7.1 months $(95 \%$ CI 5.6-8.6) and 20.1 months (95\% CI 18.4-21.8). Spearman correlation analysis showed a (very) strong positive correlation $(\rho=0.816, p<0.001)$ between TTUPc and
OS, and a moderate positive correlation $(\rho=0.471$, $p<0.001$ ) between PPS and OS (Fig. 2A/B).

\section{Impact of Reason of UTP, Pattern of Progression and Subsequent Treatment on OS and PPS}

To assess the association between the various reasons of UTP and survival, patients were categorized in 3 subgroups according to the main reason of UTP:

(A) 68 patients had radiological progression with preserved liver function and performance status,

(B) 18 patients had worsening of performance status to ECOG PS $>2$, and

(C) 27 patients developed hepatic decompensation.

Three patients had radiological progression, but data on liver function or ECOG PS were lacking. There were significant differences in OS and PPS depending on the reason of UTP (Fig. 3A, B). Patients in group A had a median OS of 20.1 months (95\% CI 18.0-22.2) compared with 12.2 (95\% CI 10.3-14.1) and 18.6 months (95\% CI 12.4-24.9) in groups $\mathrm{B}$ and $\mathrm{C}$, respectively (overall log-rank $p=0.011)$. In these subgroups, the median PPS was (A) 10.3 (95\% CI 8.0-12.6), (B) 5.3 (95\% CI 3.0-7.5) and (C) 2.4 months (95\% CI 1.7-3.2), respectively (overall logrank $p<0.001$ ). After correction for known predictors, the
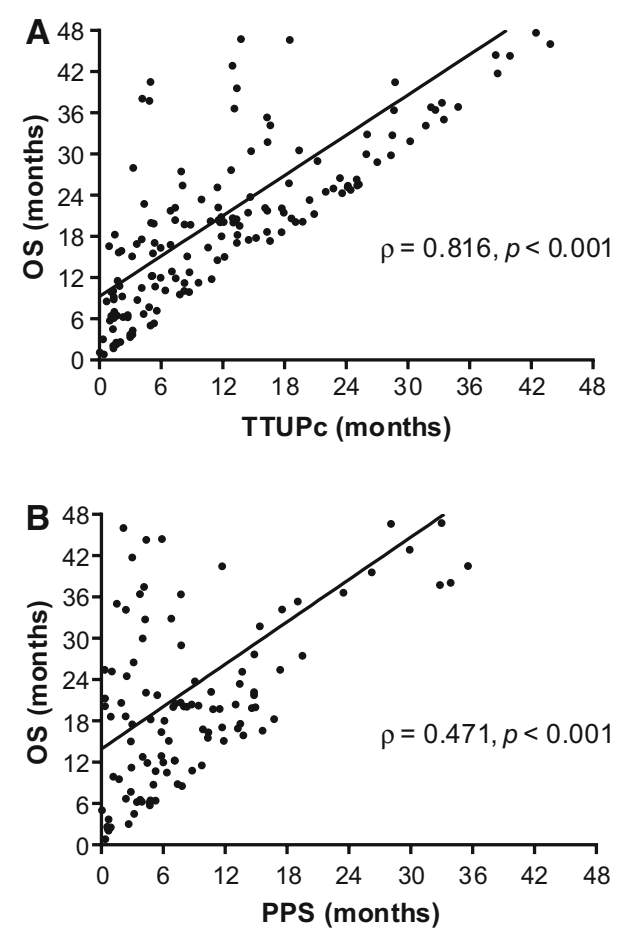

Fig. 2 Scatter plots of the correlation between overall survival (OS) and $\mathbf{A}$ time to untreatable progression (TTUPc) $\mathbf{B}$ post-progression survival (PPS) 

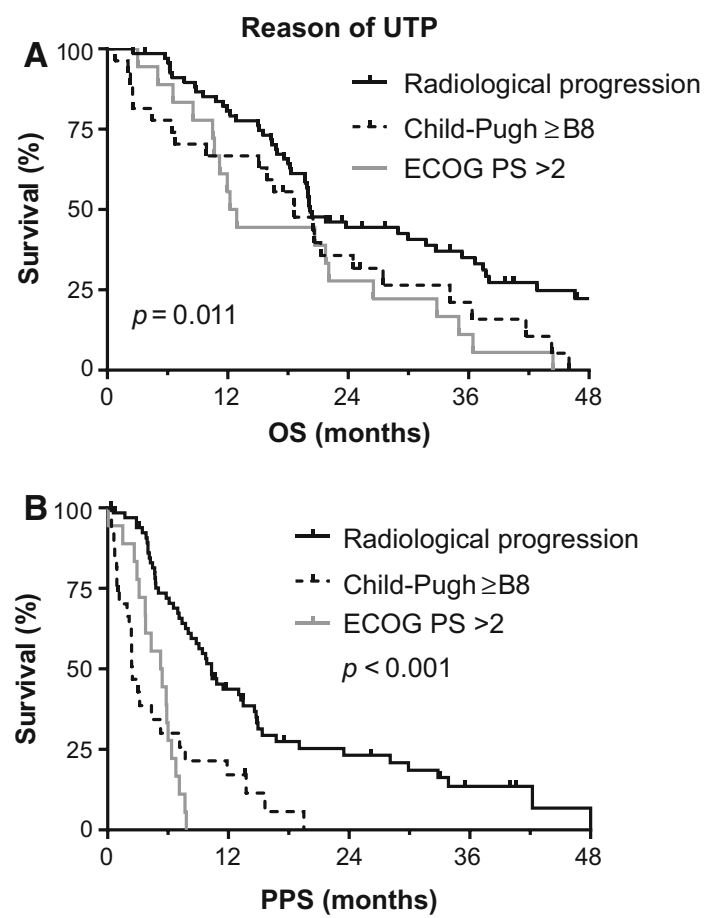

Fig. 3 A Overall survival (OS) and B post-progression survival (PPS) according to reason of unTACEable progression (UTP). ECOG PS Eastern Cooperative Oncology Group performance status reason of UTP remained an independent predictor of both OS and PPS in multivariable analysis (Tables 2 and 3).

When comparing survival between different radiological patterns of progression, there was a poorer PPS in patients with MVI (4.2 months, 95\% CI 3.3-5.2) or EHS (4.7 months, 95\% CI 3.4-6.0) compared with patients with intrahepatic progression (10.3, 95\% CI 7.8-12.9)(Fig. 4, $\log$-rank $p=0.007)$. In a subgroup analysis in patients with preserved liver function and ECOG PS $(n=68)$, MVI remained an independent predictor of PPS (HR 1.74, 95\% CI 1.74-2.85, $p=0.004$ ) (Supplementary Table 3 ). The pattern of progression in this subgroup, including $42(62 \%)$ patients with intrahepatic, $9(13 \%)$ patients with MVI and $17(25 \%)$ patients with EHS, was similar to the entire group of patients with radiological progression.

To estimate the impact of subsequent treatment on OS and PPS, we compared subgroups according to eligibility for subsequent treatment and receiving treatment or BSC only. Patients who were not eligible for subsequent treatment $(n=42)$ had the worst OS (15.9 months, 95\% CI 6.7-25.1) and PPS (3.2 months, 95\% CI 2.2-4.2). In patients eligible for subsequent treatment, receiving subsequent treatment was associated with a longer OS (21.7 vs $15.6, p=0.103)$ and PPS (13.0 vs $4.8, p=0.076)$ compared with BSC, although this was not statistically significant.
Table 2 Multivariable Cox regression analysis for overall survival

\begin{tabular}{|c|c|c|c|c|}
\hline & \multicolumn{2}{|l|}{$\begin{array}{l}\text { Whole cohort } \\
\mathrm{n}=166\end{array}$} & \multicolumn{2}{|c|}{$\begin{array}{l}\text { unTACEable progression } \\
\mathrm{n}=116\end{array}$} \\
\hline & HR [CI 95\%] & $p$ value* & HR [CI 95\%] & $p$ value* \\
\hline \multicolumn{5}{|l|}{ Prior to first TACE } \\
\hline ECOG PS 2 (Ref: $0-1)$ & $2.54(1.26-5.12)$ & 0.009 & $1.69(0.70-4.07)$ & 0.242 \\
\hline Number of nodules (Ref: 1) & Ref & - & Ref & - \\
\hline $2-3$ & $1.58(0.91-2.73)$ & 0.104 & $1.17(0.65-2.11)$ & 0.598 \\
\hline$>3 /$ diffuse & $3.04(1.71-5.40)$ & $<0.001$ & $2.02(1.07-3.81)$ & $\mathbf{0 . 0 3 1}$ \\
\hline Tumor size $>46 \mathrm{~mm}$ & $1.25(0.83-1.88)$ & 0.282 & $1.40(0.89-2.21)$ & 0.149 \\
\hline Macrovascular invasion & $2.56(1.20-5.47)$ & 0.015 & $2.44(1.00-6.00)$ & 0.051 \\
\hline $\log _{10}$ AFP & $1.51(1.26-1.82)$ & $<0.001$ & $1.59(1.31-1.94)$ & $<0.001$ \\
\hline \multicolumn{5}{|l|}{ At unTACEable progression } \\
\hline unTACEable progression & $1.88(1.06-3.32)$ & 0.031 & \multicolumn{2}{|l|}{ NA (all progressors) } \\
\hline Main reason (Ref: radiological PD) & - & - & Ref & - \\
\hline Liver dysfunction & - & - & $2.20(1.23-4.01)$ & 0.008 \\
\hline ECOG PS $>2$ & - & - & $2.09(1.10-4.00)$ & 0.025 \\
\hline
\end{tabular}

Univariable analysis shown in Supplementary Table 2

AFP Alpha-fetoprotein; CI 95 95\% confidence interval; ECOG PS Eastern Cooperative Oncology Group performance status; $H R$ hazard ratio; NA not applicable; $P D$ progressive disease; Ref reference; TACE transarterial chemoembolization

*In bold: $p<0.05$ 
Table 3 Univariable and multivariable Cox regression analysis for post-progression survival

\begin{tabular}{|c|c|c|c|c|}
\hline & \multicolumn{2}{|l|}{ Univariable } & \multicolumn{2}{|l|}{ Multivariable } \\
\hline & HR [CI 95\%] & $p$ value* & HR [CI 95\%] & $p$ value** \\
\hline \multicolumn{5}{|l|}{ Prior to TACE-1 } \\
\hline Female sex & $1.46(0.92-2.33)$ & 0.108 & & \\
\hline Age $>65$ & $1.02(0.68-1.53)$ & 0.914 & & \\
\hline HBV & $1.02(0.58-1.81)$ & 0.935 & & \\
\hline $\mathrm{HCV}$ & $1.07(0.69-1.67)$ & 0.769 & & \\
\hline Alcohol & $1.10(0.73-1.67)$ & 0.641 & & \\
\hline ECOG PS 2 (Ref: $0-1)$ & $1.70(0.81-3.58)$ & 0.160 & & \\
\hline Child-Pugh score B7 (Ref: A5-A6) & $1.51(0.73-3.14)$ & 0.267 & & \\
\hline BCLC stage (Ref: 0/A) & Ref & - & & \\
\hline $\mathrm{B}$ & $1.32(0.86-2.04)$ & 0.284 & & \\
\hline $\mathrm{C}$ & $1.82(0.80-4.16)$ & 0.153 & & \\
\hline Number of nodules (Ref: 1) & Ref & - & Ref & - \\
\hline $2-3$ & $1.36(0.82-2.26)$ & 0.238 & $1.44(0.84-2.45)$ & 0.185 \\
\hline$>3 /$ diffuse & $1.62(0.96-2.74)$ & 0.071 & $1.21(0.66-2.21)$ & 0.544 \\
\hline Tumor size $>46 \mathrm{~mm}$ & $0.83(0.56-1.25)$ & 0.372 & & \\
\hline Macrovascular invasion & $1.53(0.71-3.32)$ & 0.280 & & \\
\hline $\log _{10}$ AFP & $1.30(1.10-1.53)$ & 0.003 & $1.36(1.14-1.62)$ & 0.001 \\
\hline \multicolumn{5}{|l|}{ At unTACEable progression } \\
\hline unTACEable progression & \multicolumn{2}{|c|}{ NA (all progressors) } & \multicolumn{2}{|c|}{ NA (all progressors) } \\
\hline Reason (Ref: radiological PD) & Ref & - & Ref & - \\
\hline Liver dysfunction & $3.10(1.88-5.11)$ & $<0.001$ & $3.24(1.82-5.74)$ & $<0.001$ \\
\hline ECOG PS $>2$ & $3.74(2.07-6.75)$ & $<0.001$ & $3.83(2.09-7.01)$ & $<0.001$ \\
\hline
\end{tabular}

*In bold: included in multivariable analysis $(p$ value $<0.1)$

**In bold: $p$ value $<0.05$

$A F P$ Alpha-fetoprotein; BCLC Barcelona Clinic Liver Cancer classification; $C I 9595 \%$ confidence interval; $E C O G$ PS Eastern Cooperative Oncology Group performance status; $H B V$ hepatitis B virus; $H C V$ hepatitis $\mathrm{C}$ virus; $\mathrm{HR}$, hazard ratio; NA not applicable; $P D$ progressive disease; Ref reference; TACE transarterial chemoembolization

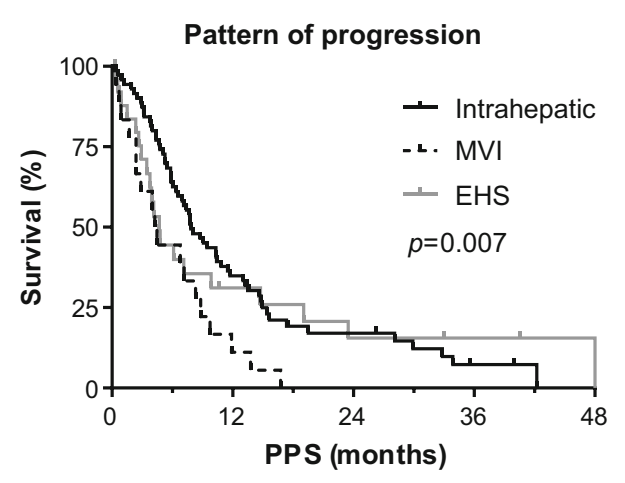

Fig. 4 Post-progression survival (PPS) according to radiological pattern of progression. EHS extrahepatic spread; $M V I$ macrovascular invasion

\section{Discussion}

In this study of HCC patients treated with TACE, the majority of patients (58\%) had preserved liver function and ECOG PS at UTP. These patients showed a trend toward better PPS when treated with subsequent liver-directed or systemic treatment compared with BSC only (13 vs 5 months). These results are in line with post hoc analyses of the landmark phase III sorafenib trials and a recent international observational study [27-29], showing a clear survival benefit in strictly selected patients treated with sorafenib after TACE compared with those receiving BSC only [29]. Although sorafenib is the guideline-recommended treatment strategy after TACE failure [1], the predominantly intrahepatic pattern of progression at UTP 
(75\%) implies that most patients may be candidate for both liver-directed (radioembolization) and systemic treatments (sorafenib). Future studies might provide predictive markers that can guide the choice for radioembolization or sorafenib in order to maximize survival benefit of patients with UTP.

Although a minority of patients discontinued TACE because of hepatic decompensation $(23 \%)$ or deteriorated ECOG PS (18\%), these factors were independent factors for OS and PPS in our study. This underscores the need for assessment of liver function and ECOG PS after TACE treatment. The prognostic importance of these parameters in patients with HCC is widely accepted, reflected by its implementation in the BCLC staging system [1, 2]. Still, this is the first study to quantify its prognostic impact in context of switching from TACE to subsequent treatments. Prior studies reported 0-60\% hepatic decompensation after TACE $[3,5,6,13,30-32]$, depending on patient selection and the definition of hepatic decompensation. In our study, only 3 out of $27(11 \%)$ patients who developed hepatic decompensation following TACE recovered enough to receive subsequent treatment. This may indicate that hepatic decompensation following is caused by a severe underlying liver disease or an aggressive tumor biology compromising liver function. These patients have a poor prognosis and are unlikely to benefit from the currently available subsequent treatments. In future studies, nonliver metabolized treatment options, i.e., immunotherapy, may be considered.

In concordance with a prior Japanese study [22], we showed that OS correlated strongly with TTUPc in HCC patients treated with TACE. This validates TTUPc as a novel surrogate endpoint for OS in both European and Japanese patients. Common surrogate endpoints in oncology such as time to progression (TTP) and progression-free survival (PFS) have limited accuracy in representing TACE success by not taking into account 'reTACEable' progression or the competing risk of underlying liver cirrhosis [15]. Therefore, current guidelines do not recommend TTP or PFS as endpoints in HCC [1]. TTUPc showed a strong correlation with OS and has the advantage of requiring a significantly shorter follow-up than OS (median of 13 vs 22 months), making it an interesting endpoint for future clinical trials in HCC investigating TACE or new treatments combined with TACE. Because patients are increasingly receiving multiple lines of anti-HCC treatment following TACE failure, this will result in a prolonged post-TACE survival (PPS) diluting the effect of TACE on OS. This highlights the value of TTUPc as a validated surrogate marker for TACE benefit.

Limitations to our study include the retrospective study design with its inherent drawbacks. Still, our study is representable for the multidisciplinary management of
HCC patients undergoing TACE in a European country, with an OS that is similar to prior studies [26].

In conclusion, our data suggest that most patients discontinue TACE due to intrahepatic tumor progression with preserved ECOG PS and liver function, making them potential candidates for subsequent liver-directed or systemic treatment. Hepatic decompensation or deteriorated ECOG PS was independently associated with poor OS and PPS. TTUPc correlated strongly with OS, making this a potential surrogate endpoint for future trials estimating TACE benefit.

Acknowledgements We thank Mrs. N.S. Mostafavi for her advice on the statistical analysis.

Funding This study was not supported by any funding.

\section{Compliance with Ethical Standards}

Conflict of interest Heinz-Josef Klümpen is a member of the advisory board for Ipsen and Sirtex and received an unrestricted research grant from Bayer (no grant numbers apply). Bart Takkenberg, served as a speaker for Gore WL, Bayer and Norgine, is a member of the advisory board for Gilead and has received grants from the Netherlands Organisation for Health Research and Development (ZonMw) and The Netherlands Society for Gastroenterology (Gastrostart). Otto van Delden served as a consultant for Cook Medical. Tim Labeur declared no conflicts of interest. The study was designed and conducted by academic investigators.

Ethical Approval All procedures performed in studies involving human participants were in accordance with the ethical standards of the institutional and/or national research committee and with the 1964 Declaration of Helsinki and its later amendments or comparable ethical standards. For this type of study, formal consent is not required.

Informed Consent This study has obtained IRB approval from the Amsterdam UMC, and the need for informed consent was waived.

Consent for Publication For this type of study, consent for publication is not required.

Open Access This article is distributed under the terms of the Creative Commons Attribution 4.0 International License (http:// creativecommons.org/licenses/by/4.0/), which permits unrestricted use, distribution, and reproduction in any medium, provided you give appropriate credit to the original author(s) and the source, provide a link to the Creative Commons license, and indicate if changes were made.

\section{References}

1. Galle PR, Forner A, Llovet JM, Mazzaferro V, Piscaglia F, Raoul $\mathrm{J}$-L, et al. EASL clinical practice guidelines: management of hepatocellular carcinoma. J Hepatol. 2018;69(1):182-236.

2. Vogel A, Cervantes A, Chau I, Daniele B, Llovet J, Meyer T, et al. Hepatocellular carcinoma: ESMO Clinical Practice Guidelines for diagnosis, treatment and follow-up. Ann Oncol. 2018;29(Suppl 4):iv238-55. 
3. Lo CM, Ngan H, Tso WK, Wong J. Randomized controlled trial of transarterial Lipiodol chemoembolization for unresectable hepatocellular carcinoma. Hepatology. 2002;35:1164-71.

4. Llovet JM, Real MI, Montaña X, Planas R, Coll S, Aponte J, et al. Arterial embolisation or chemoembolisation versus symptomatic treatment in patients with unresectable hepatocellular carcinoma: a randomised controlled trial. Lancet. 2002;359(9319):1734-9.

5. Camma C, Schepis F, Orlando A, Albanese M, Shahied L, Trevisani $\mathrm{F}$, et al. Transarterial chemoembolization for unresectable hepatocellular carcinoma: meta-analysis of randomized controlled trials. Radiology. 2002;224(1):47-54.

6. Llovet JM, Bruix J. Systematic review of randomized trials for unresectable hepatocellular carcinoma: chemoembolization improves survival. Hepatology. 2003;37:429-42.

7. Llovet JM, Ricci S, Mazzaferro V, Hilgard P, Gane E, Blanc JF, et al. Sorafenib in advanced hepatocellular carcinoma. N Engl J Med. 2008;359(4):378-90.

8. Kudo M, Finn RS, Qin S, Han KH, Ikeda K, Piscaglia F, et al. Lenvatinib versus sorafenib in first-line treatment of patients with unresectable hepatocellular carcinoma: a randomised phase 3 non-inferiority trial. Lancet. 2018;391(10126):1163-73.

9. Bruix J, Qin S, Merle P, Granito A, Huang YH, Bodoky G, et al. Regorafenib for patients with hepatocellular carcinoma who progressed on sorafenib treatment (RESORCE): a randomised, double-blind, placebo-controlled, phase 3 trial. Lancet. 2016;389:56-66.

10. Abou-Alfa GK, Meyer T, Cheng A-L, El-Khoueiry AB, Rimassa $\mathrm{L}$, Ryoo B-Y, et al. Cabozantinib $(\mathrm{C})$ versus placebo $(\mathrm{P})$ in patients (pts) with advanced hepatocellular carcinoma (HCC) who have received prior sorafenib: results from the randomized phase III CELESTIAL trial. J Clin Oncol. 2018;36(Suppl 4):207.

11. Chau I, Peck-Radosavljevic M, Borg C, Malfertheiner P, Seitz JF, Park JO, et al. Ramucirumab as second-line treatment in patients with advanced hepatocellular carcinoma following first-line therapy with sorafenib: patient-focused outcome results from the randomised phase III REACH study. Eur J Cancer. 2017;81:17-25.

12. El-Khoueiry AB, Sangro B, Yau T, Crocenzi TS, Kudo M, Hsu $\mathrm{C}$, et al. Nivolumab in patients with advanced hepatocellular carcinoma (CheckMate 040): an open-label, non-comparative, phase $1 / 2$ dose escalation and expansion trial. Lancet. 2017;389(10088):2492-502.

13. Hépatocellulaire GdEedTdC. A comparison of lipiodol chemoembolization and conservative treatment for unresectable hepatocellular carcinoma. N Engl J Med. 1995;332(19):1256-61.

14. Evaluation of Sorafenib in Combination With Local Microtherapy Guided by Gd-EOB-DTPA Enhanced MRI in Patients With Inoperable Hepatocellular Carcinoma [updated 2 December 2016. https://clinicaltrials.gov/ct2/show/study/NCT01126645. Accessed 30 Aug 2018.

15. Bruix J, Reig M, Rimola J, Forner A, Burrel M, Vilana R, et al. Clinical decision making and research in hepatocellular carcinoma: pivotal role of imaging techniques. Hepatology. 2011;54(6):2238-44.

16. Kudo M, Matsui O, Izumi N, Kadoya M, Okusaka T, Miyayama $\mathrm{S}$, et al. Transarterial chemoembolization failure/refractoriness: JSH-LCSGJ criteria 2014 pdate. Oncology. 2014;87(Suppl 1):22-31.

17. Raoul JL, Gilabert M, Piana G. How to define transarterial chemoembolization failure or refractoriness: a European perspective. Liver cancer. 2014;3(2):119-24.

18. Reig M, Rimola J, Torres F, Darnell A, Rodriguez-Lope C, Forner A, et al. Postprogression survival of patients with advanced hepatocellular carcinoma: rationale for second-line trial design. Hepatology. 2013;58(6):2023-31.

19. Douhara A, Namisaki T, Moriya K, Kitade M, Kaji K, Kawaratani $\mathrm{H}$, et al. Predisposing factors for hepatocellular carcinoma recurrence following initial remission after transcatheter arterial chemoembolization. Oncol Lett. 2017;14(3):3028-34.

20. Park W, Chung YH, Kim JA, Jin YJ, Lee D, Shim JH, et al. Recurrences of hepatocellular carcinoma following complete remission by transarterial chemoembolization or radiofrequency therapy: focused on the recurrence patterns. Hepatol Res. 2013;43(12):1304-12.

21. Rou WS, Lee BS, Moon HS, Lee ES, Kim SH, Lee HY. Risk factors and therapeutic results of early local recurrence after transcatheter arterial chemoembolization. World J Gastroenterol. 2014;20(22):6995-7004.

22. Arizumi T, Ueshima K, Iwanishi M, Minami T, Chishina $H$, Kono M, et al. The overall survival of patients with hepatocellular carcinoma correlates with the newly defined time to progression after transarterial chemoembolization. Liver Cancer. 2017;6(3):227-35.

23. Bruix J, Reig M, Sherman M. Evidence-based diagnosis, staging, and treatment of patients with hepatocellular carcinoma. Gastroenterology. 2016;150(4):835-53.

24. Lammer J, Malagari K, Vogl T, Pilleul F, Denys A, Watkinson A, et al. Prospective randomized study of doxorubicin-eluting-bead embolization in the treatment of hepatocellular carcinoma: results of the PRECISION V study. Cardiovasc Intervent Radiol. 2010;33(1):41-52.

25. Xie ZB, Wang XB, Peng YC, Zhu SL, Ma L, Xiang BD, et al. Systematic review comparing the safety and efficacy of conventional and drug-eluting bead transarterial chemoembolization for inoperable hepatocellular carcinoma. Hepatol Res. 2015;45(2):190-200.

26. Raoul JL, Sangro B, Forner A, Mazzaferro V, Piscaglia F, Bolondi L, et al. Evolving strategies for the management of intermediate-stage hepatocellular carcinoma: available evidence and expert opinion on the use of transarterial chemoembolization. Cancer Treat Rev. 2011;37(3):212-20.

27. Bruix J, Raoul JL, Sherman M, Mazzaferro V, Bolondi L, Craxi A, et al. Efficacy and safety of sorafenib in patients with advanced hepatocellular carcinoma: subanalyses of a phase III trial. J Hepatol. 2012;57(4):821-9.

28. Cheng AL, Guan Z, Chen Z, Tsao CJ, Qin S, Kim JS. Efficacy and safety of sorafenib in patients with advanced hepatocellular carcinoma according to baseline status: subset analyses of the phase III sorafenib Asia-Pacific trial. Eur J Cancer. 2012;48(10): 1452-65.

29. Yen C, Sharma R, Rimassa L, Arizumi T, Bettinger D, Choo HY, et al. Treatment stage migration maximizes survival outcomes in patients with hepatocellular carcinoma treated with sorafenib: an observational study. Liver Cancer. 2017;6(4):313-24.

30. Burrel M, Reig M, Forner A, Barrufet M, de Lope CR, Tremosini $S$, et al. Survival of patients with hepatocellular carcinoma treated by transarterial chemoembolisation (TACE) using drug eluting beads. Implications for clinical practice and trial design. J Hepatol. 2012;56(6):1330-5.

31. Garwood ER, Fidelman N, Hoch SE, Kerlan RK Jr, Yao FY. Morbidity and mortality following transarterial liver chemoembolization in patients with hepatocellular carcinoma and synthetic hepatic dysfunction. Liver Transplant. 2013;19(2):164-73.

32. Llovet JM. Arterial embolization or chemoembolization versus symptomatic treatment in patients with unresectable hepatocellular carcinoma: a randomised controlled trial. Lancet. 2002;359:1734-9. 